\title{
A convenient synthesis of trans -3-hydroxy-L-proline
}

\author{
Surajit Sinha, Santosh Tilve, ${ }^{+}$and Srinivasan Chandrasekaran ${ }^{*}$ \\ Department of Organic Chemistry, Indian Institute of Science, Bangalore-560012, India \\ E-mail:scn@orgchem.iisc.ernet.in
}

\section{Dedicated to Professor S. Swaminathan on his $\mathbf{8 0}^{\text {th }}$ birthday}

(received 03 Jan 05; accepted 16 Feb 05; published on the web 02 Mar 05)

\begin{abstract}
A simple synthesis of trans-3-hydroxy-L-proline has been achieved starting from $\beta$ alanine making use of Sharpless asymmetric epoxidation as a key step in the synthesis.
\end{abstract}

Keywords: $\beta$-Alanine, Sharpless asymmetric epoxidation, hydroxyproline

\section{Introduction}

3-Hydroxyprolines are important components and chiral synthons for biologically active compounds such as cyclothialidine, ${ }^{1}$ mucrorin- $\mathrm{D},{ }^{2}$ telomycin ${ }^{3}$ and polyhydroxylated alkaloids ${ }^{4}$. Trans-3-hydroxy-L-proline 1 was isolated from bovine Achilles tendon collagen ${ }^{5}$. Several syntheses of 3-hydroxy prolines in general ${ }^{6}$ and trans-3-hydroxy-L-proline in particular ${ }^{7}$ have been reported. Most of the methods utilized enzymatic methods or chiron approach to get the optically pure product.

We decided to study a new approach to the asymmetric synthesis of trans-3-hydroxy-Lproline 1 using Sharpless asymmetric epoxidation as a key step in the synthetic strategy. A retrosynthetic analysis of this approach is depicted in scheme 1.

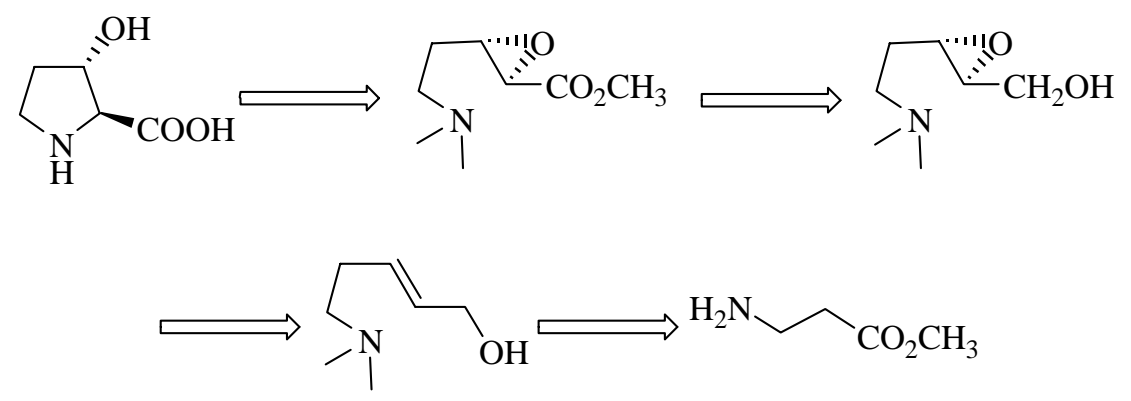

Scheme 1. Retrosynthetic analysis for compound 1. 
trans-3-Hydroxy proline can be derived by an intramolecular cyclisation of an amino epoxide. The chiral epoxide can be generated by Sharpless epoxidation of a suitable allylic alcohol. The allylic alcohol in turn can be derived from $\beta$-alanine by straightforward synthetic manipulations (Scheme 1).

The synthetic route was started with the readily available and inexpensive $\beta$-alanine. A suitable protective group for the amino group was needed which will be stable to Sharpless epoxidation conditions later in the sequence. It is well documented in the literature ${ }^{8}$ that the tertbutoxycarbonyl or benzyloxycarbonyl protected amines usually lead to the formation of cyclic urethane derivatives under these conditions. Various type of N-protecting groups that would encourage cyclization through nitrogen, namely $N$-tosyl, $N$-trityl, and even unprotected derivatives have been tried but in all the cases the reaction leads to a complex mixture of products. All the problems are taken care of when nitrogen is protected both with tosyl as well as benzyl group. ${ }^{9}$

The synthetic route adopted to achieve the synthesis of $\mathbf{1}$ is presented in Scheme 2. The methyl ester of $\beta$-alanine was readily converted to the $N$-protected methylester 2 , which on reduction with lithium aluminium hydride in ether $\left(0^{\circ} \mathrm{C}\right)$ provided the alcohol 3 in quantitative yield. The alcohol 3 was then oxidized with Dess-Martin periodinane to afford the aldehyde $\mathbf{4}$ in $97 \%$ yield, while the Swern oxidation of the same compound gave the product only in $69 \%$ yield. The aldehyde $\mathbf{4}$ was allowed to react with triethylphosphono acetate ${ }^{10}$ and the E-olefinic ester $\mathbf{5}$ was obtained in $89 \%$ yield. Initially the reduction of the unsaturated ester $\mathbf{5}$ was tried with lithium aluminium hydride in ether but it led to a mixture of allylic alcohol $\mathbf{6}$ and the corresponding saturated alcohol. However, when the reduction of $\mathbf{5}$ was carried out with $\mathrm{AlH}_{3}$, generated in situ, the allylic alcohol $\mathbf{6}$ was the only product obtained in quantitative yield.

Now the stage was set for carrying out Sharpless asymmetric epoxidation. The allyl alcohol 6 was treated with titanium tetraisopropoxide, tert-butyl hydroperoxide, and L-(+)-diethyl tartarate $^{11}$ and the epoxy alcohol 7 was obtained in 92\% yield with $98 \%$ e.e. The enantiomeric excess was estimated by NMR shift reagent $\left[\mathrm{Eu}(\mathrm{hfc})_{3}\right]$ experiments.

Initially, the direct oxidation of the epoxy alcohol 7 to the epoxy acid 8 with $\mathrm{RuCl}_{3} / \mathrm{NaIO}_{4}{ }^{12}$ was carried out but the yield obtained in this reaction was generally poor (45\% of corresponding methyl ester). Therefore, the epoxy alcohol 7 was first oxidized to the aldehyde with DessMartin periodinane, followed by oxidation with $\mathrm{Ag}_{2} \mathrm{O}$ to get the epoxy carboxylic acid 8 (91\%). Compound $\mathbf{8}$ was converted to its methyl ester $\mathbf{9}$ on treatment with methyl iodide in high yield. NMR experiments with chiral shift reagent $\left[\mathrm{Eu}(\mathrm{hfc})_{3}\right]$ revealed the product $\mathbf{9}$ to have optical purity of $98 \%$ e.e. 

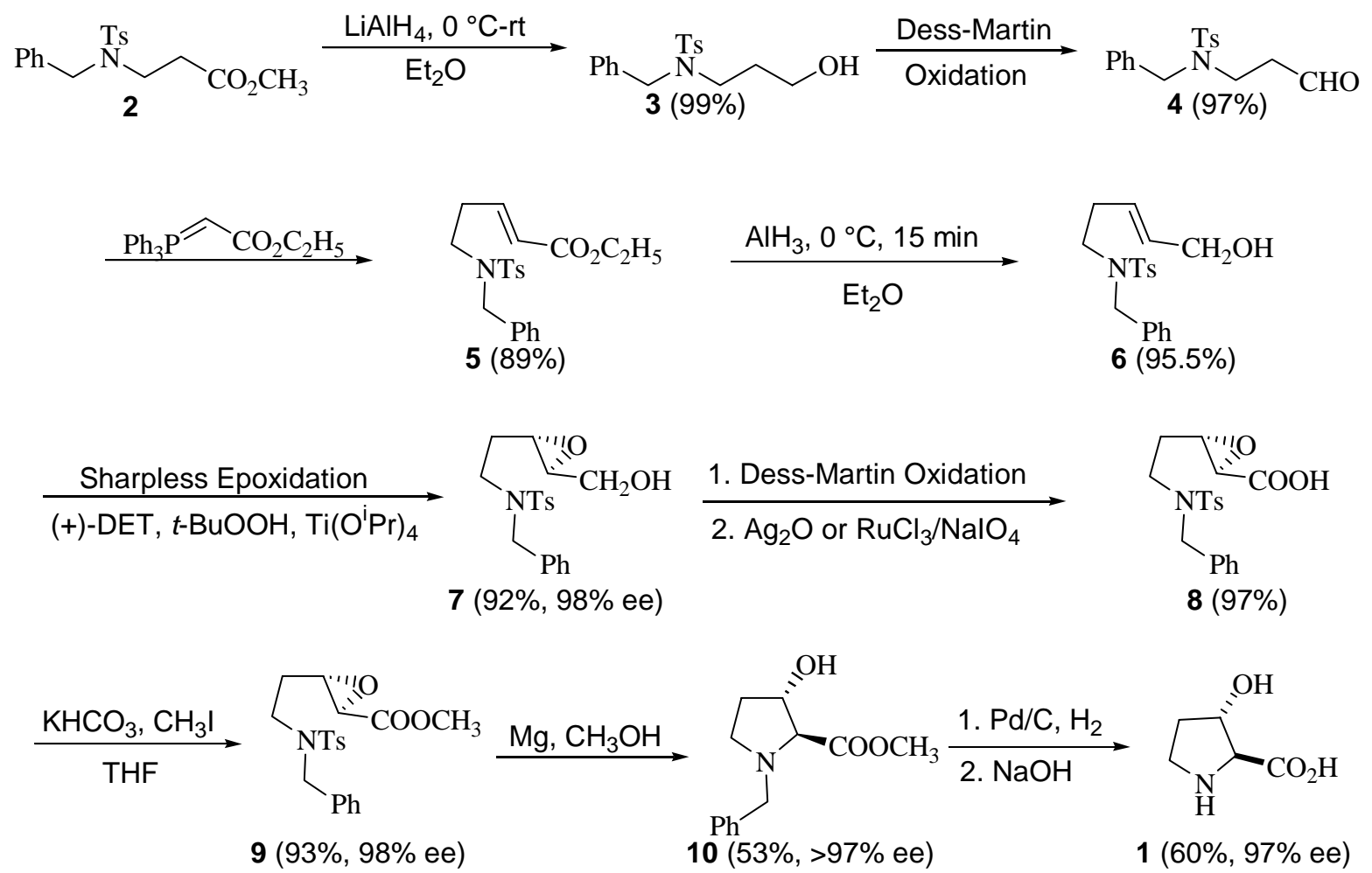

Scheme 2. Synthesis of trans-3-hydroxy-L-proline.

It was then decided to deprotect the N-benzyl group, which then can be cyclized on treatment with $\mathrm{K}_{2} \mathrm{CO}_{3} / \mathrm{MeOH}$. Unfortunately, the reaction of compound 9 with $\mathrm{Pd} / \mathrm{C} / \mathrm{H}_{2}$ did not effect the hydrogenolysis as desired. Even under forcing conditions at higher hydrogen pressure the hydrogenolysis could not be accomplished efficiently. The next strategy was to remove the tosyl protective group of $\mathbf{9}$ with $\mathrm{Na}$ /napthalene. ${ }^{9}$ Here again, the reaction was not very clean. Finally detosylation of $\mathbf{9}$ was carried out successfully by reacting it with $\mathrm{Mg} / \mathrm{MeOH} .{ }^{13}$ Under these conditions, not only did the tosyl group undergo cleavage but it also underwent in situ cyclisation to give the $N$-benzylated 3-hydroxy-L-proline derivative 10 (33\% overall yield from $\beta$-alanine; based on recovered starting material).The ${ }^{1} \mathrm{H}$ NMR of $\mathbf{1 0}$ did not give any indication as to whether it was the cis- or the trans- isomer. However, it was found to be a single diastereomer with $97 \%$ e.e. as confirmed by NMR chiral shift reagent $\left[\mathrm{Eu}(\mathrm{hfc})_{3}\right]$ experiment as well as HPLC analysis on a chiral column [cyclodex $\alpha$-pm, solvent: $10 \%$ water containing $1 \%$ tetraethyl ammonium acetate buffer in $\mathrm{MeOH}$, flow rate 0.4, retention time $5.99 \mathrm{~min}]$. For purposes of characterization, compound $\mathbf{1 0}$ was converted to the corresponding para-toluene sulfonate salt. In the ${ }^{1} \mathrm{H}$ NMR of this salt, the $\alpha-\mathrm{H}$ appeared as a clear doublet at $\delta 3.18$ with a coupling constant of $1.7 \mathrm{~Hz}$ indicating it to be the trans product. Compound $\mathbf{1 0}$ on hydrogenolysis $\left(\mathrm{Pd} / \mathrm{C} / \mathrm{H}_{2}\right)$ followed by saponification gave a residue which was purified by resin bead column chromatography (elution with $1.5-2 \mathrm{M}$ aq. $\mathrm{NH}_{3}$ solution) to afford $\mathbf{1}$ as a white solid with the melting point $\left(228-236^{\circ} \mathrm{C}\right)\left(\right.$ lit. $\left.^{\mathrm{7a}, 7 \mathrm{~b}} 228-235^{\circ} \mathrm{C}\right)$ and optical rotation $[\alpha]=-18.3^{\circ}\left\{\right.$ lit. $^{7}[\alpha]=-$ 
18.8 data identical to those, reported in the literature for trans-3-hydroxy-L-proline. Its optical purity was found to be $>97 \%$ e.e.

In conclusion it has been demonstrated that starting from inexpensive achiral $\beta$-alanine, the 13-step synthesis of enantiopure trans-3-hydroxy-L-proline 1 has been achieved using Sharpless asymmetric epoxidation as a key-step.

\section{Experimental Section}

Preparation of $\mathbf{T s B n N C H}{ }_{2} \mathrm{CH}_{2} \mathrm{CH}_{2} \mathrm{OH}$ (3). To a stirred solution of TsBnNCH $\mathrm{CH}_{2} \mathrm{CO}_{2} \mathrm{Me}_{2}$ $(15 \mathrm{~g}, 0.04 \mathrm{~mol})$ in anhydrous $\mathrm{Et}_{2} \mathrm{O}(150 \mathrm{~mL})$, lithium aluminum hydride $(2 \mathrm{~g}, 0.052 \mathrm{~mol})$ was added at $0^{\circ} \mathrm{C}$ over a period of $30 \mathrm{~min}$ and the reaction mixture was stirred at that temperature for another $30 \mathrm{~min}$. The reaction mixture was quenched with moist $\mathrm{Et}_{2} \mathrm{O}$ and filtered through a pad of Celite. The organic layer was washed with $1 \mathrm{~N} \mathrm{HCl}(2 \times 40 \mathrm{~mL})$, brine, dried over anhydrous $\mathrm{Na}_{2} \mathrm{SO}_{4}$. The solvent was removed under reduced pressure. The product 3 was obtained as a colorless liquid after column chromatography on silica gel (13.78 g, 99\%).

IR (neat, $\mathrm{cm}^{-1}$ ): 3500-3300; 1590; 1450; 1490; 1320; 1150; ${ }^{1} \mathrm{H} \mathrm{NMR}\left(\mathrm{CDCl}_{3}, 90 \mathrm{MHz}\right) \delta: 1.40$ (m, $2 \mathrm{H},-\mathrm{CH}_{2} \underline{\mathrm{C}}_{2} \mathrm{CH}_{2-}$ ); 1.60 (br s, $1 \mathrm{H},-\mathrm{OH}$ ); 2.40 (s, $3 \mathrm{H}, \mathrm{Ar}_{-} \underline{\mathrm{C}}_{3}$ ); 3.20 (t, J=6 Hz, $2 \mathrm{H}$, $\mathrm{C}_{2}{ }_{2} \mathrm{~N}-$ ); 3.50 (t, $J=6 \mathrm{~Hz}, 2 \mathrm{H},-\underline{\mathrm{C}}_{2} \mathrm{O}-$ ); 4.25 (s, $2 \mathrm{H},-\underline{\mathrm{C}}_{2} \mathrm{Ph}$ ); 7.25 (m, 7H, aromatic protons); $7.709 \mathrm{~d}, J=7.7 \mathrm{~Hz}, 2 \mathrm{H}$, aromatic protons).

Preparation of $\mathbf{T s B n N C H}_{2} \mathrm{CH}_{2} \mathbf{C H O}$ (4). Procedure A. Anhydrous DMSO (2.7 mL, 37.60 mmol) was added drop-wise to oxalyl chloride in anhydrous $\mathrm{CH}_{2} \mathrm{Cl}_{2}(40 \mathrm{~mL})$ at $-60^{\circ} \mathrm{C}$ under argon. It was stirred for $5 \mathrm{~min}$ and then the alcohol 3 (2 g, $6.26 \mathrm{mmol})$ in $\mathrm{CH}_{2} \mathrm{Cl}_{2}(10 \mathrm{~mL})$ was added over a period of $15 \mathrm{~min}$. The reaction mixture was stirred for another half an hour $\left(-60^{\circ} \mathrm{C}\right)$ after which $\mathrm{Et}_{3} \mathrm{~N}$ (5.5 mL, $40 \mathrm{mmol}$ ) was added dropwise and it was allowed to warm to room temperature $\left(25^{\circ} \mathrm{C}\right)$ over a period of $1 \mathrm{~h}$. The reaction mixture was washed with $1 \mathrm{~N} \mathrm{HCl} \mathrm{(15}$ $\mathrm{mL}$ ), brine $(25 \mathrm{~mL})$ and dried over anhydrous $\mathrm{Na}_{2} \mathrm{SO}_{4}$. The solvent was removed in vacuo. After purification by column chromatography on silica gel (using 15\% EtOAc in hexane as eluent), the pure aldehyde 4 was obtained as a colorless liquid (1.37 g, 69\%).

Procedure B. Oxidation of alcohol 3 with Dess Martin periodinane. To a stirred solution of periodinane $(4.0 \mathrm{~g}, 8.4 \mathrm{mmol})$ in $\mathrm{CH}_{2} \mathrm{Cl}_{2}(15 \mathrm{~mL})$ was added a solution of alcohol 3 (2.0 g, $6.26 \mathrm{mmol})$ in $\mathrm{CH}_{2} \mathrm{Cl}_{2}(10 \mathrm{~mL})$. Within $5 \mathrm{~min}$, a white precipitate appeared. The heterogeneous reaction mixture was stirred for $40 \mathrm{~min}$. It was diluted with diethyl ether $(75 \mathrm{~mL})$ and the resulting suspension of iodinane was added to $1.3 \mathrm{M} \mathrm{NaOH}(30 \mathrm{~mL})$. After the mixture was stirred for $10 \mathrm{~min}$, the ether layer was washed successively with $1.3 \mathrm{M} \mathrm{NaOH}(30 \mathrm{~mL})$ solution, and brine $(20 \mathrm{~mL})$, dried over anhydrous sodium sulfate and concentrated to give the crude product. This, on purification on a silica gel column gave the pure aldehyde $\mathbf{4}$ as a colorless oil (1.93 g, 97\%). 
IR (neat, $\left.\mathrm{cm}^{-1}\right): 1690 ; 1600 ; 1440 ; 1330 .{ }^{1} \mathrm{H} \mathrm{NMR}\left(\mathrm{CDCl}_{3}, 90 \mathrm{MHz}\right) \delta: 2.40$ (s, 3H, $\left.-\mathrm{ArC}_{3}\right)$; 2.60 (t, $\left.J=6 \mathrm{~Hz}, 2 \mathrm{H},-\underline{\mathrm{C}}_{2} \mathrm{CHO}\right) ; 3.40$ (t, $\left.J=6 \mathrm{~Hz}, 2 \mathrm{H},-\underline{\mathrm{C}}_{2} \mathrm{~N}-\right) ; 4.20$ (s, 2H, $\left.-\underline{\mathrm{H}}_{2} \mathrm{Ph}\right) ; 7.30$ (m, $7 \mathrm{H}$, aromatic protons); 7.75 (d, $J=7.7 \mathrm{~Hz}, 2 \mathrm{H}$, aromatic protons); 9.90 (s, $1 \mathrm{H},-\mathrm{CHO}$ ). EIMS: 317( $\left.\mathrm{M}^{+}\right)$.

Preparation of (E)- $\mathbf{T s B n N C H} \mathrm{CH}_{2} \mathbf{C H}=\mathrm{CHCO}_{2}$ Et (5). The mixture of aldehyde 4 (0.242 g, $0.76 \mathrm{mmol}), \mathrm{K}_{2} \mathrm{CO}_{3}(0.21 \mathrm{~g}, 1.52 \mathrm{mmol})$, triphenylphosphon-acetate $(182 \mu \mathrm{L}, 0.91 \mathrm{mmol})$ and water $(0.16 \mathrm{~mL})$ was stirred for $16 \mathrm{~h}$ at room temperature. EtOAc $(10 \mathrm{~mL})$ was added and the reaction mixture was washed with water $(5 \mathrm{~mL})$, and brine, dried over anhydrous $\mathrm{Na}_{2} \mathrm{SO}_{4}$, filtered, and the solvent was removed under reduced pressure. Purification by column chromatography on silica gel (using 10\% EtOAc in hexane as eluent) yielded $\mathbf{5}$ as a colorless liquid (0.262 g, 89\%).

IR (neat, $\left.\mathrm{cm}^{-1}\right): 1720 ; 1590 ; 1490 ; 1450 ; 1325 ; 1150 .{ }^{1} \mathrm{H} \mathrm{NMR}\left(\mathrm{CDCl}_{3}, 300 \mathrm{MHz}\right) \delta: 1.26(\mathrm{t}, J=$ $\left.6.9 \mathrm{~Hz}, 3 \mathrm{H},-\mathrm{OCH}_{2} \mathrm{CH}_{3}\right) ; 2.23\left(\mathrm{~m}, 2 \mathrm{H},-\underline{\mathrm{CH}}_{2} \mathrm{CH}=\right)$; 2.45 (s, $\left.3 \mathrm{H}, \mathrm{ArC}_{3}\right) ; 3.18$ (t, $J=7.5 \mathrm{~Hz}, 2 \mathrm{H}$, $\mathrm{NC}_{2_{2}}$ ); 4.16 (q, $\left.J=6.9 \mathrm{~Hz}, 2 \mathrm{H},-\mathrm{OC}_{2}{ }_{2} \mathrm{CH}_{3}\right) ; 4.30$ (s, 2H, - $\left.\underline{\mathrm{H}}_{2} \mathrm{Ph}\right) ; 5.62$ (dd, $J_{1}=15.45 \mathrm{~Hz}, J_{2}$ $=1.6 \mathrm{~Hz}, 1 \mathrm{H},-\mathrm{CH}=\mathrm{C} \underline{\mathrm{HCO}}-) ; 6.64$ (dt, $\left.J_{1}=15.6 \mathrm{~Hz}, J_{2}=7.2 \mathrm{~Hz}, 1 \mathrm{H},-\mathrm{CH}_{2} \mathrm{CH}=\mathrm{CH}-\right) ; 7.30$ (m, $7 \mathrm{H}$, aromatic protons); 7.73 (d, $J=8 \mathrm{~Hz}, 2 \mathrm{H}$, aromatic protons). LRMS m/z (\%): 386 [( $\left.\mathrm{M}^{+}-1\right)$, 1.4]; 364 (4.4); 342 (22); 274 (100); 181 (16); 155 (24); 92 (100). Elemental Analysis: Calculated for $\mathrm{C}_{21} \mathrm{H}_{25} \mathrm{O}_{4} \mathrm{SN}$ : $\mathrm{C}=65.11 ; \mathrm{H}=6.46$; $\mathrm{N}=3.62$; Found: $\mathrm{C}=64.92 ; \mathrm{H}=6.31 ; \mathrm{N}=3.54$.

Preparation of (E)-TsBnNCH $\mathbf{C H}_{2} \mathbf{C H}=\mathbf{C H C H} \mathbf{C H}_{2} \mathrm{OH}$ (6). $\mathrm{AlCl}_{3}$ (4.1 g, $0.031 \mathrm{~mol}$ ) was added portion wise over a period of $20 \mathrm{~min}$ to a stirred solution of lithium aluminum hydride (4.1 g, $0.11 \mathrm{~mol})$ in $\mathrm{Et}_{2} \mathrm{O}(300 \mathrm{~mL})$ at $0^{\circ} \mathrm{C}$. It was stirred for $5 \mathrm{~min}$ and the ester 5 (10.5 g, $\left.0.027 \mathrm{~mol}\right)$ in $\mathrm{Et}_{2} \mathrm{O}(80 \mathrm{~mL})$ was added drop-wise through a dropping funnel $(10 \mathrm{~min})$ at $0^{\circ} \mathrm{C}$. Then the reaction mixture was quenched with moist $\mathrm{Et}_{2} \mathrm{O}$. The organic layer was separated and the aqueous layer was washed with $\mathrm{Et}_{2} \mathrm{O}(50 \mathrm{~mL})$. The combined organic layer was washed with $2 \mathrm{~N}$ $\mathrm{HCl}(100 \mathrm{~mL})$, brine $(60 \mathrm{~mL})$ and dried over anhydrous $\mathrm{Na}_{2} \mathrm{SO}_{4}$. The solvent was removed in vacuo. Purification by column chromatography on silica gel (using 30\% EtOAc in hexane as eluent) yielded the allylic alcohol 6 as a colorless liquid (8.9 g, 95.5\%).

IR (neat, $\left.\mathrm{cm}^{-1}\right)$ : 3400-3200; 1600; 1445; 1325; ${ }^{1} \mathrm{H}$ NMR $\left(\mathrm{CDCl}_{3}, 300 \mathrm{MHz}\right) \delta: 1.65$ (br s, $1 \mathrm{H},-$ $\mathrm{OH}$ ); 2.05 (m, 2H, $-\mathrm{CH}_{2} \mathrm{C}_{2} \mathrm{CH}=$ ); 2.44 (s, $\left.3 \mathrm{H}, \mathrm{Ar}-\underline{\mathrm{C}}_{3}\right) ; 3.15$ (t, J = $\left.7.5 \mathrm{~Hz}, 2 \mathrm{H},-\mathrm{NC}_{2} \underline{2}^{-}\right) ; 3.95$

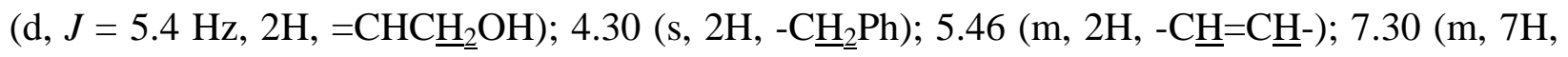
aromatic protons); 7.72 (d, $J=8.4 \mathrm{~Hz}, 2 \mathrm{H}$, aromatic protons); LRMS (m/z, \%): 344 [( $\left.\left.\mathrm{M}^{+}-1\right), 5\right]$; 274 (100); 258 (8); 181 (80); 155 (29); 106 (29); 91 (100).

\section{Procedure for Sharpless epoxidation of allylic alcohol (6)}

A two neck round bottomed flask (500 $\mathrm{mL}$ ) equipped with a magnetic stirring bar, and molecular sieves ( $0.700 \mathrm{~g}$, powder), was oven dried, then fitted with a septum and flushed with argon. The flask was charged with anhydrous $\mathrm{CH}_{2} \mathrm{Cl}_{2}(200 \mathrm{~mL})$ and cooled to $-24^{\circ} \mathrm{C}$. Then the following liquids were added sequentially via syringe: titanium tetraisopropoxide $(7.55 \mathrm{~mL}, 25 \mathrm{mmol}), \mathrm{L}-$ 
(+)-diethyl tartrate ( $4.5 \mathrm{~mL}, 26.5 \mathrm{mmol}$ ), allyl alcohol 6 (7.3 g, $21 \mathrm{mmol}$, the mixture was stirred for $5 \mathrm{~min}$ before the addition of 6) and finally tert- butyl hydroperoxide (12.6 mL, $63 \mathrm{mmol}, 3$ eq, 5 molar solution in toluene). The resulting homogeneous solution was then stirred for $22 \mathrm{~h}$ at $-24^{\circ} \mathrm{C}$. Aqueous tartaric acid solution $(10 \% 65 \mathrm{~mL})$ was added while stirring and, after $30 \mathrm{~min}$, the cooling bath was removed and stirring was continued at room temperature for approximately $1 \mathrm{~h}$ until the aqueous layer became clear. After separation of the aqueous layer, the organic layer was washed once with water [M14], dried over anhydrous sodium sulfate, filtered, and concentrated to afford a colorless oil with an odor revealing contamination by tert-butyl hydroperoxide. This oil was diluted with ether $(150 \mathrm{~mL})$, the resulting solution was cooled in an ice bath, and then $1 N$ sodium hydroxide solution $(70 \mathrm{~mL}$ ) was added. This produced a two-phase mixture which was stirred at $0^{\circ} \mathrm{C}$ for $30 \mathrm{~min}$. The ether layer was washed with brine, dried over anhydrous sodium sulfate, and concentrated under reduced pressure. After purification by column chromatography on silica gel (using 30\% EtOAc in hexane as eluent), the pure epoxy alcohol 7 was obtained as a colorless viscous liquid $(7 \mathrm{~g}, 92 \%)$. [ $[\alpha]_{D}{ }^{25}=-9$ (c $=1$ in $\mathrm{CH}_{2} \mathrm{Cl}_{2}$ ). IR (neat, $\left.\mathrm{cm}^{-1}\right)$ : 3500-3200; 1590; 1490; 1440; 1320; 1150. ${ }^{1} \mathrm{H}$ NMR $\left(\mathrm{CDCl}_{3}, 300 \mathrm{MHz}\right) \delta: 1.55$ (2H, m, $-\mathrm{CH}_{2} \mathrm{C}_{2} \mathrm{CH}(\mathrm{O})$ )); 1.88 (br s, $-\mathrm{OH}$ ); 2.45 (s, 3H, $\mathrm{ArC}_{3}$ ); 2.80 (m, $2 \mathrm{H},-\mathrm{NC}_{2} \mathrm{CH}_{2}$ ); 3.25 (m, 2H, - $\left.\mathrm{CH}(\mathrm{O}) \mathrm{C}_{2} \mathrm{OH}\right) ; 3.65$ (m, $\left.1 \mathrm{H},-\mathrm{CH}_{2} \mathrm{C} \underline{\mathrm{H}}(\mathrm{O})-\right)$; 3.72 (m, $\left.1 \mathrm{H},-\mathrm{C} \underline{\mathrm{H}}(\mathrm{O}) \mathrm{CH}_{2} \mathrm{OH}\right) ; 4.30$ (s, $\left.2 \mathrm{H},-\mathrm{C}_{2} \mathrm{Ph}\right) ; 7.30$ (m, 7H, aromatic protons); 7.70 (d, $J=8.0 \mathrm{~Hz}, 2 \mathrm{H}$, aromatic protons); ${ }^{13} \mathrm{C}$ NMR $\left(\mathrm{CDCl}_{3}, 75 \mathrm{MHz}\right) \delta: 21.2 ; 30.5 ; 45.1 ; 51.9 ; 52.4 ; 58.0 ; 61.5 ; 126.8 ; 127.8 ; 128.1 ; 128.3$; 129.5; 135.9; 143.3; LRMS (m/z, \%): 359 [( $\left.\left(\mathrm{M}^{+}-2\right), 0.50\right] ; 343$ (0.70); 274 (80); 206 (100); 181 (7); 155 (10); 91 (100). Elemental analysis: Calculated for $\mathrm{C}_{19} \mathrm{H}_{23} \mathrm{O}_{4} \mathrm{SN}$ : $\mathrm{C}=63.16, \mathrm{H}=6.37$, $\mathrm{N}=3.88$; Found: $\mathrm{C}=62.89, \mathrm{H}=6.64, \mathrm{~N}=4.01$.

\section{Conversion of epoxy alcohol 7 to epoxy ester 9}

To a stirred solution of periodinane (2 g, $4.21 \mathrm{mmol})$ in $\mathrm{CH}_{2} \mathrm{Cl}_{2}(15 \mathrm{~mL})$ was added a solution of alcohol $7(1.16 \mathrm{~g}, 3.21 \mathrm{mmol})$ in $\mathrm{CH}_{2} \mathrm{Cl}_{2}(10 \mathrm{~mL})$. Within 5 min a white precipitate appeared. The heterogeneous reaction mixture was stirred for $40 \mathrm{~min}$, diluted with ether $(75 \mathrm{~mL})$ and the resulting suspension of iodinane was added to $1.3 \mathrm{M} \mathrm{NaOH}(30 \mathrm{~mL})$. After the mixture was stirred for $10 \mathrm{~min}$, the ether layer was washed with $1.3 \mathrm{M} \mathrm{NaOH}(30 \mathrm{~mL})$ solution, and brine $(20$ $\mathrm{mL}$ ), dried over anhydrous sodium sulfate and filtered. Removal of the solvent gave the corresponding the aldehyde as a colorless oil (1.15 g, 99.8\%).

Silver nitrate $(1.6 \mathrm{~g}, 9.5 \mathrm{mmol})$ in water $(40 \mathrm{~mL})$ was added to well stirred solution of sodium hydroxide $(0.750 \mathrm{~g}, 18.75 \mathrm{mmol})$ in water $(40 \mathrm{~mL})$. A solution of the crude epoxy aldehyde (obtained as described above) (1.15 g, $3.20 \mathrm{mmol})$ in THF (10 mL) was added drop-wise to the suspension and stirred at room temperature for $3 \mathrm{~h}$. The residue was filtered through a pad of Celite and washed with water $(5 \mathrm{~mL})$. After washing with ether $(15 \mathrm{~mL})$, the aqueous layer was acidified with cold $1 N \mathrm{HCl}$ and the white precipitate was extracted with ether $(2 \times 30 \mathrm{~mL})$. The organic layer was dried over anhydrous $\mathrm{Na}_{2} \mathrm{SO}_{4}$ and concentrated under reduced pressure to yield the carboxylic acid $\mathbf{8}$ as a colorless liquid (1.17 g, 97\%). 
IR (neat, $\mathrm{cm}^{-1}$ ): 3400-2800; 1720; 1600; 1450; 1330; 1150. ${ }^{1} \mathrm{H}$ NMR $\left(\mathrm{CDCl}_{3}, 300 \mathrm{MHz}\right) \delta: 1.50$ (m, $\left.1 \mathrm{H},-\mathrm{CH}_{2} \mathrm{C} \underline{\mathrm{H}}(\mathrm{H}) \mathrm{CH}(\mathrm{O})-\right) ; 1.80$ (m, $\left.1 \mathrm{H},-\mathrm{CH}_{2} \mathrm{C} \underline{\mathrm{H}}(\mathrm{H}) \mathrm{CH}(\mathrm{O})-\right) ; 2.46$ (s, 3H, $\left.\mathrm{ArC}_{3}\right)$; 3.0 (m, $\left.1 \mathrm{H},-\mathrm{CH}_{2} \mathrm{C} \underline{\mathrm{H}}(\mathrm{H}) \mathrm{N}-\right)$; 3.10 (d, $\left.J=2.1 \mathrm{~Hz}, 1 \mathrm{H},-\mathrm{CH} 2 \mathrm{C} \underline{\mathrm{H}}(\mathrm{O})-\right)$; 3.25 (m, $\left.1 \mathrm{H},-\mathrm{CH}_{2} \mathrm{C} \underline{\mathrm{H}}(\mathrm{H}) \mathrm{N}-\right)$; 4.30 (s, $2 \mathrm{H},-\mathrm{C}_{2} \mathrm{Ph}$ ); 7.30 (m, 7H, aromatic protons); 7.75 (d, $J=8.7 \mathrm{~Hz}, 2 \mathrm{H}$, aromatic protons).

Methyl iodide $(0.4 \mathrm{~mL}, 6.24 \mathrm{mmol})$ was added to a stirred mixture of crude epoxy carboxylic acid 8 (1.17 g, $3.12 \mathrm{mmol})$ and $\mathrm{KHCO}_{3}(0.406 \mathrm{~g}, 4 \mathrm{mmol})$ in anhydrous DMF $(8 \mathrm{~mL})$. The reaction mixture was stirred at room temperature for $12 \mathrm{~h}$. DMF was removed under reduced pressure, $\mathrm{Et}_{2} \mathrm{O}(50 \mathrm{~mL})$ was added to the residue and the mixture was washed with water $(10 \mathrm{~mL})$, sodium thiosulfate solution $(10 \mathrm{~mL}, 20 \%)$, and brine. The organic phase was dried over anhydrous sodium sulfate, filtered, and the filtrate was evaporated in vacuo. After purification by column chromatography on silica gel (using 20\% EtOAc in hexane as eluent), the pure epoxy ester 9 was obtained as a colorless liquid (1.13 g, 93\%). Overall yield of 9 from alcohol 7 was $91 \%$.

$[\alpha]_{D}^{25}=-23.34\left(\mathrm{c}=1.5\right.$ in $\mathrm{CH}_{2} \mathrm{Cl}_{2}$ ). IR (neat, $\left.\mathrm{cm}^{-1}\right)$ : 1730; 1590; 1500; 1460; 1340; 1200; 1150 . ${ }^{1} \mathrm{H} \quad \mathrm{NMR}\left(\mathrm{CDCl}_{3}, 300 \mathrm{MHz}\right) \delta: 1.56\left(\mathrm{~m}, 1 \mathrm{H},-\mathrm{CH}_{2} \mathrm{C} \underline{\mathrm{H}}(\mathrm{H}) \mathrm{CH}(\mathrm{O})-\right) ; 1.65(\mathrm{~m}, 1 \mathrm{H},-$ $\left.\mathrm{CH}_{2} \mathrm{C} \underline{\mathrm{H}}(\mathrm{H}) \mathrm{CH}(\mathrm{O})-\right) ; 2.45$ (s, 3H, $\left.\mathrm{ArC}_{3}\right) ; 2.98$ (m, 1H, $\left.-\mathrm{CH}_{2} \mathrm{C} \underline{\mathrm{H}}(\mathrm{H}) \mathrm{N}-\right)$; 3.07 (d, $J=1.8 \mathrm{~Hz}, 1 \mathrm{H}$,

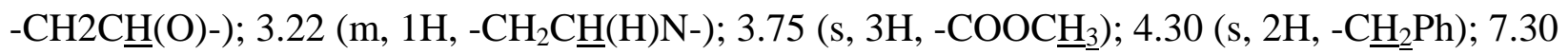
(m, $7 \mathrm{H}$, aromatic protons); $7.72\left(\mathrm{~d}, J=8.4 \mathrm{~Hz}, 2 \mathrm{H}\right.$, aromatic protons); ${ }^{13} \mathrm{C}$ NMR $\left(\mathrm{CDCl}_{3}\right.$, $100 \mathrm{MHz}) \delta$ : 20.6; 30.1; 4.1; 5.9; 52.1; 52.6; 55.0; 126.4; 127.2; 127.6; 127.9; 129.0; 135.3; 135.5; 142.7; 168.3. LRMS (m/z, \%): 274 [(TsBnN $\left.\left.{ }^{+}=\mathrm{CH}_{2}\right), 15\right] ; 234$ [( $\left.\left.{ }^{+}-\mathrm{Tos}\right), 12\right] ; 155$ (2); 118 (4); 91 (100). Elemental analysis: Calculated for $\mathrm{C}_{20} \mathrm{H}_{23} \mathrm{O}_{5} \mathrm{NS}$ : $\mathrm{C}=61.69, \mathrm{H}=5.91, \mathrm{~N}=3.60$. Found: $\mathrm{C}=61.42, \mathrm{H}=5.83, \mathrm{~N}=3.46$.

\section{Detosylation followed by cyclisation of 9 with $\mathrm{Mg} / \mathrm{MeOH}$}

To a suspension of $\mathrm{Mg}(0.388 \mathrm{~g}, 16 \mathrm{mmol})$ in anhydrous $\mathrm{MeOH}(40 \mathrm{~mL})$ was added a solution of the epoxy ester $9(0.91 \mathrm{~g}, 2.35 \mathrm{mmol})$ in anhydrous $\mathrm{MeOH}(20 \mathrm{~mL})$. The resulting suspension was stirred at room temperature for $12 \mathrm{~h}$. $\mathrm{MeOH}$ was removed in vacuo and the residue was diluted with $\mathrm{CHCl}_{3}(60 \mathrm{~mL})$ and filtered through a pad of Celite ${ }^{\circledR}$. The $\mathrm{CHCl}_{3}$ layer was washed with brine, dried over anhydrous sodium sulfate and concentrated under reduced pressure. After purification by column chromatography on silica gel (using MeOH: tert-BuOMe: hexane 0.5: 2: 3.5 as eluent), proline derivative 10 was obtained as a colorless liquid $(0.293 \mathrm{~g}, 53 \%)$; $[\alpha]_{D}^{25}=-$ 22.1 ( $\mathrm{c}=0.6$ in $\mathrm{CH}_{2} \mathrm{Cl}_{2}$ ). Starting material $9(0.110 \mathrm{~g}, 31 \%$ ) was recovered from the reaction. IR (neat, $\left.\mathrm{cm}^{-1}\right)$ : 3400-3100; 1730; 1450; 1200; ${ }^{1} \mathrm{H}$ NMR $\left(\mathrm{CDCl}_{3}, 300 \mathrm{MHz}\right) \delta: 1.75(\mathrm{~m}, 1 \mathrm{H},-$ $\mathrm{C} \underline{\mathrm{H}}(\mathrm{H}) \mathrm{CH}(\mathrm{OH})-) ; 1.95$ (m, 1H, $-\mathrm{C} \underline{\mathrm{H}}(\mathrm{H}) \mathrm{CH}(\mathrm{OH})-) ; 2.0$ (br s, $-\mathrm{OH}) ; 2.80$ (t, $J=6.6 \mathrm{~Hz}, 2 \mathrm{H},-$ $\left.\mathrm{C}_{2}{ }_{2} \mathrm{~N}-\right) ; 3.27$ (m, $\left.1 \mathrm{H},-\mathrm{CH}_{2} \mathrm{CH}(\mathrm{OH})-\right)$; 3.28 (d, $\left.J=1.8 \mathrm{~Hz}, 1 \mathrm{H},-\mathrm{C} \underline{\mathrm{H}}\left(\mathrm{COOCH}_{3}\right) \mathrm{N}-\right)$; 3.77 (s, $3 \mathrm{H},-$ $\left.\mathrm{COOC}_{3}\right) ; 3.80$ (s, $\left.2 \mathrm{H},-\underline{\mathrm{C}}_{2} \mathrm{Ph}\right) ; 7.30$ (m, $5 \mathrm{H}$, aromatic protons). ${ }^{1} \mathrm{H}$ NMR $\left(\mathrm{CDCl}_{3}+\mathrm{D}_{2} \mathrm{O}, 300\right.$ MHz) $\delta: 1.75$ (m, 1H); 1.95 (m, 1H); 2.82 (t, $J=6.6 \mathrm{~Hz}, 2 \mathrm{H}) ; 3.27$ (m, 1H); 3.28 (d, $J=1.8 \mathrm{~Hz}$, $\left.{ }^{1} \mathrm{H}\right) ; 3.78$ (s, 3H); 3.80 (s, 2H); 7.30 (m, 5H). LRMS (m/z, \%): 236 [( $\left.\mathrm{M}^{+}+1\right)$, (3.5)]; 176 (8); 146 (100); 117 (5); 91 (100); 65 (14); Elemental Analysis: Calculated for $\mathrm{C}_{20} \mathrm{H}_{25} \mathrm{O}_{6} \mathrm{SN}$ : C 58.97, $\mathrm{H}$ 6.14, N 3.44. Found C 58.89, H 6.29, N 3.60. 
Data for $p$-toluene sulfonate salt of 10: $\mathrm{mp}: 151-153^{\circ} \mathrm{C}$.

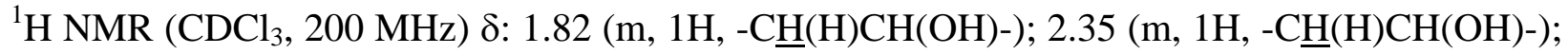
2.40 (s, $\left.3 \mathrm{H}, \mathrm{ArC}_{3}\right)$; 2.95 (t, $\left.J=7.8 \mathrm{~Hz}, 2 \mathrm{H},-\underline{\mathrm{C}}_{2} \mathrm{~N}-\right)$; 3.14 (m, $\left.1 \mathrm{H},-\mathrm{CH}_{2} \mathrm{CH}(\mathrm{OH})-\right)$; 3.18 (d, $J=$ $\left.1.7 \mathrm{~Hz}, 1 \mathrm{H},-\mathrm{C} \underline{\mathrm{H}}\left(\mathrm{COOCH}_{3}\right) \mathrm{N}-\right) ; 3.73$ (s, 3H, $\left.-\mathrm{COOCH}_{3}\right) ; 4.13$ (s, 2H, $\left.-\underline{\mathrm{C}}_{2} \mathrm{Ph}\right) ; 7.35$ (m, 7H, aromatic protons); 7.70 (d, $J=8.1 \mathrm{~Hz}, 2 \mathrm{H}$, aromatic protons); 9.14 (br s, -N $\left.\underline{\mathrm{H}}\left(\mathrm{CH}_{2} \mathrm{Ph}\right)-\right)$.

${ }^{1} \mathrm{H}$ NMR $\left(\mathrm{CDCl}_{3}+\mathrm{D}_{2} \mathrm{O}, 200 \mathrm{MHz}\right) \delta: 1.90(\mathrm{~m}, 1 \mathrm{H}) ; 2.25(\mathrm{~m}, 1 \mathrm{H}) ; 2.38(\mathrm{~s}, 3 \mathrm{H}) ; 2.95(\mathrm{t}, J=$ $7.8 \mathrm{~Hz}, 2 \mathrm{H}) ; 3.14(\mathrm{~m}, 1 \mathrm{H}) ; 3.20$ (d, $J=1.7 \mathrm{~Hz}, 1 \mathrm{H}) ; 3.73$ (s, 3H); 4.14 (s, 2H); 7.35 (m, 7H); $7.70(\mathrm{~d}, J=8.1 \mathrm{~Hz}, 2 \mathrm{H})$.

\section{Preparation of trans-3-hydroxy-L-proline (1)}

A mixture of the hydroxy ester 10 (0.045 g, $0.19 \mathrm{mmol})$ and $10 \% \mathrm{Pd}-\mathrm{C}(16 \mathrm{mg})$ in $15 \% \mathrm{HCO}_{2} \mathrm{H}$ in $\mathrm{MeOH}(4 \mathrm{~mL})$ was hydrogenated at room temperature for $24 \mathrm{~h}$ under an atmosphere of hydrogen. The catalyst was filtered off and the solvent was evaporated under reduced pressure to give the debenzylated product as a brown oil. This crude product was taken up in a mixture of $\mathrm{MeOH}(0.6 \mathrm{~mL})$ and aq. $2 \mathrm{~N} \mathrm{NaOH}(200 \mu \mathrm{L})$ and stirred at room temperature for $4 \mathrm{~h}$. Then the reaction mixture was acidified with $1 \mathrm{~N} \mathrm{HCl}$ to $\mathrm{pH} \sim 2$ and the solvent was removed in vacuo. The residue was dissolved in water $(1 \mathrm{~mL})$ and purified by ion-exchange chromatography [Dowex resin, 1.5-2M aq. $\mathrm{NH}_{3}$ solution]. After evaporation of the solvent the trans-3-hydroxy-L-proline 1 was obtained as a colorless solid $(0.015 \mathrm{~g}, 60 \%) . \mathrm{mp}$ : $\left(228-236^{\circ} \mathrm{C}\right.$ decomp.) (lit. $\left.{ }^{7} 228-235^{\circ} \mathrm{C}\right)$; $[\alpha]_{\mathrm{D}}{ }^{25}-18.3\left(\mathrm{c}-0.2, \mathrm{H}_{2} \mathrm{O}\right)$; lit. $^{7}[\alpha]_{\mathrm{D}}{ }^{20}-18.8$

IR (KBr): 3410, 2920; ${ }^{1} \mathrm{H}-\mathrm{NMR}\left(\mathrm{D}_{2} \mathrm{O}, 300 \mathrm{MHz}\right) \delta: 2.0$ (2H, m, - $\left.\underline{\mathrm{H}}_{2} \mathrm{CH}(\mathrm{OH})-\right), 3.4(1 \mathrm{H}, \mathrm{m},-$ C$\left.\left(\mathrm{CH}_{2}\right) \mathrm{NH}-\right)$, 3.5 (1H, m, - $\left.\underline{\mathrm{H}}\left(\mathrm{CH}_{2}\right) \mathrm{NH}-\right)$, 4.1(1H, s, - $\left.\underline{\mathrm{H}}(\mathrm{OH})-\right), 4.6(1 \mathrm{H}, \mathrm{m},-\mathrm{CH}(\mathrm{COOH}) \mathrm{NH}-)$; EIMS: $131\left(\mathrm{M}^{+}\right)$.

\section{Acknowledgements}

The authors thank the Department of Science and Technology, New Delhi for financial support of this investigation.

\section{References and Notes}

$+\quad$ SERC-DST Visiting Fellow on leave from Goa University.

* Presented in the Electronic Conference on Synthetic Organic Chemistry (ECSOC-4, 2000).

1. Nakada, N.; Shimada, H.; Hirata, T.; Aoki, Y.; Kamiyama, T.; Watanabe, J.; Arisawa, M. Antimicrobial Agents and Chemotherapy 1993, 37, 2656.

2. Tschesche, R.; Samuel, T.D.; Uhlendorf, J.; Fehlaber, H.W. Chem. Ber. 1972 105, 3106.

3. Sheehan, J.C.; Whitney, J.G. J. Am. Chem. Soc. 1962, 84, 3980. 
4. (a) Sardina, F.J.; Rapoport, H. Chem. Rev. 1996, 96, 1825. (b) Fleet, G.W.J.; Son, J.C. Tetrahedron 1998, 44, 2637.

5. Ogle, J.; Arlinghaus, R.; Logan, M. Arch. Biochem. Biophysics 1961, 94, 85.

6. (a) Morita, K.; Irreverre, F.; Sakiyama, F.; Witkop, B. J. Am. Chem. Soc. 1963, 85, 2832. (b) Sheehan, J.C.; Whitney, J.G. J. Am. Chem. Soc. 1963, 85, 3863. (c) Wolff, III, J.S.; Ogle, J.D.; Logan, M.A. J. Biol. Chem. 1996, 241, 1300. (d) Cooper, J.; Gallagher, P.T.; Knight, D.W., J. Chem. Soc., Perkin Trans. 1 1993, 1313. (e) Jurczak, J.; Prokopowicz, P.; Golebiowski, A.; Tetrahedron Lett. 1993, 7107. (f) Sundram, H.; Golebiowski, A.; Johnson, C.R.; Tetrahedron Lett. 1994, 6975. (g) Sibi, M.P.; Christensen, J.W. Tetrahedron Lett. 1995, 6213. (h) Dell’Uomo, N.; Di Giovanni, M.C.; Misiti, D.; Zappia, G.; Monache, G.D. Tetrahedron: Asymmetry 1996, 7, 181. (i) Gotschi, E.; Jenny, C-J.; Reindl, P.; Ricklin, F. Helv. Chim. Acta. 1996, 79, 2219.

7. (a) Herdeis, C.; Hubmann, H.P. Tetrahedron: Asymmetry 1994, 5, 119. (b) Lee, J.H.; Kang, J.E.; Yang, M.S.; Kang, K.Y.; Park, K.H. Tetrahedron 2001, 57, 10071.

8. Baldwin, J.E.; Flinn, A. Tetrahedron Lett. 1987, 3605.

9. Adams, C.E.; Walker, F.J.; Sharpless, K.B. J. Org. Chem. 1985, 50, 422.

10. Villieras, J.; Rambaudet, M.; Graff, M. Tetrahedron Lett. 1985, 53.

11. Pfenninger, A. Synthesis 1986, 89.

12. Carlsen, P.J.; Katsuki, T.; Martin, V.S.; Sharpless, K.B. J. Org. Chem. 1981, 46, 3937.

13. Alonso, D.A.; Andersson, P. G. J. Org. Chem. 1998, 63, 9455. 